\title{
CORRECTION
}

\section{Correction: Phenotypic expansion of CACNA1C-associated disorders to include isolated neurological manifestations}

Lance H. Rodan, Rebecca C. Spillmann, Harley T. Kurata, Shawn M. Lamothe, Jasmine Maghera, Rami Abou Jamra, Anna Alkelai, Stylianos E. Antonarakis, Isis Atallah, Omer Bar-Yosef, Frédéric Bilan, Kathrine Bjorgo, Xavier Blanc, Patrick Van Bogaert, Yoav Bolkier, Lindsay C. Burrage, Björn U. Christ, Jorge L. Granadillo, Patricia Dickson, Kirsten A. Donald, Christèle Dubourg, Aviva Eliyahu, Lisa Emrick, Kendra Engleman, Michaela Veronika Gonfiantini, Jean-Marc Good, Judith Kalser, Chiara Kloeckner, Guus Lachmeijer, Marina Macchiaiolo, Francesco Nicita, Sylvie Odent, Emily O'Heir, Xilma Ortiz-Gonzalez, Marta Pacio-Miguez, María Palomares-Bralo, Loren Pena, Konrad Platzer, Mathieu Quinodoz, Emmanuelle Ranza, Jill A. Rosenfeld, Eliane Roulet-Perez, Avni Santani, Fernando Santos-Simarro, Ben Pode-Shakked, Cara Skraban, Rachel Slaugh, Andrea Superti-Furga, Isabelle Thiffault, Richard H. van Jaabrsveld, Marie Vincent, Hong-Gang Wang, Pia Zacher and Undiagnosed Diseases Network*, Eric Rush, Geoffrey S. Pitt, Ping Yee Billie Au and Vandana Shashi iD

Genetics in Medicine (2021) 23:2016; https://doi.org/10.1038/s41436-021-01306-7

Correction to: Genetics in Medicine 2021; https://doi.org/10.1038/ s41436-021-01232-8; published online 23 June 2021
In the prior version of this article the author name Geoffrey S. Pitt was incorrectly written as Geoffrey Pitt. The original article has been corrected. 